\title{
Scanning Electron Microscopy and Analysis of Moist, Wet and Liquid Specimens
}

\author{
P. Echlin.
}

Cambridge Analytical Microscopy, Cambridge, CB4 1XA, United Kingdom.

Water is an integral constituent of our planet and a ubiquitous solvent of most of the solid, liquid and gas phases that make up the earths surface. Nearly all metals, inorganic dry samples such as rocks, dry organic samples such as wood, polymers, biological materials and mud, paint, food and drink contain varying amounts of water and, in some cases, organic fluids. Conventional scanning electron microscopy and microanalysis demands that water and organic fluids must either be removed or immobilized before they may be examined and analyses. Alternatively, the scanning microscope can be modified to accommodate these types of material in the microscope column. Details of sample stabilization and charge elimination will not be considered here .

The more usual (and convenient procedure) is to first dry the sample before it is exposed to the arid environment of the microscope column. Drying metals and hard robust inorganic materials is a relatively straightforward procedure provided the drying process does not compromise the structural and chemical integrity of the specimen. More attention has to be paid when drying robust organic material and polymers and biological and wet specimens require even more attention. This paper will briefly consider the ways available to accommodate these more demanding samples.

The first approach is to modify the scanning electron microscope and do nothing to the samples. The earliest attempt to look at wet and moist specimens was to place them in a discreet chamber maintained as close as possible to the natural environment of the specimen, which was placed in the microscope column. These wet cells have undergone considerable development and are now available commercially. The very small wet and moist specimens can be examined by backscattered and secondary imaging. An alternate approach is to use variable-pressure scanning electron microscopes. In these instruments, the specimen chamber is separated from the column by a pressure-limiting aperture, which allow specimens to be examined in their natural state at pressures of $10-200 \mathrm{pA}$. A pressure of $200 \mathrm{pA}$ and $295 \mathrm{~K}$ is the saturated pressure of water. These microscopes allow wet, moist and liquid sample to be imaged and analyzed. These two approaches are providing a lot of new information and the only disadvantage at the moment is that they do not provide high resolution images and there remain problems concerning radiation damage and Brownian movement within the specimens which are undergoing analysis.

The second approach is to make no changes to the microscope but to make chemical changes to the specimen. These methods centre on removing the liquids, principally water, by dissolving them in a variety of organic liquids using a number of different techniques. Wet and moist specimen may be dehydrated by gradually dissolving the water in increasingly concentration of organic fluids such as ethanol. These are familiar and well-established methods that are used to remove water from 
biological samples. The speed of dehydration may be fast or slow and carried out at varying temperatures but in all cases the dehydrated specimen ends up in a pool of pure alcohol. Such specimens need further processing before they can be examined and analyses in the microscope. One popular way forward is to use critical point drying. This procedure is based on the principle that there is a critical point on the isotherm for many liquids, such as carbon dioxide, where the density of the liquid and gaseous phase, are identical. At this critical temperature-pressure point there is an equal exchange of molecules between the gas and liquid phases and surface tension is zero. This popular method overcomes the problems associated with air drying samples. In such samples, as the water/air interface passes initially through the surface and then the bulk of the drying specimen, the surface tensions forces associated with the interface can rise to as high as 20-100M.N (2000psi). This dramatic increase in surface tension will severely distort the structural integrity of soft sample. The advantages of these chemical procedures are that they are simple and effective. The disadvantages are that they may cause spatially unequal dimensional changes, selective erosion and perturbation of surface features and selective removal of chemical components.

The third approach is to make no changes to the microscope and only make physical changes to the specimen such as freeze-drying and freeze substitution. Freeze drying is an effective physical dehydration method by which water (ice) is removed by low temperature sublimation in a high vacuum. The advantage of freeze-drying is that it only involves changing water from the liquid $>$ solid $>$ vapor phase. Freeze substitution is a chemical dehydration process in which the ice in a frozen hydrated system is dissolved in an organic solvent at low temperatures which, in turn, are replaced with an organic polymer. The advantage's of these two methods are that they both very effectively remove water from specimens and, provided the initial quench cooling is sufficiently fast, there is excellent retention of structural and chemical integrity. The disadvantage is that only very small, i.e. tens of micrometers, samples can be prepared for high-resolution images and analysis.

The final approach is to simply rapidly cool the sample and examine and analyze it at low temperatures on a cold stage in the scanning electron microscope. This approach retains the high quality imaging and analytical features of the microscope on specimens, which have only undergone a phase change from the liquid to the solid state. But even this approach is not ideal. At high spatial resolution, there are molecular and structural artifact and the fully frozen specimens are very sensitive to radiation damage. On the other hand, it is the only minimally invasive procedure that retain the sample as close as possible to its natural structure and chemical identity.

More detailed information and practical protocols for these approaches to preparing these seemingly intractable specimens, may be found in a recent publication (1).

\section{References}

[1] P. Echlin, "Handbook of Sample Preparation for Scanning Electron Microscopy and X-Ray Microanalysis" Springer, New York. (2009). 